\title{
Introducción al modelado de información de construcción (BIM) en edificaciones e infraestructuras de ingeniería
}

\section{Introduction to building information modeling (BIM) in buildings and engineering infrastructure}

\author{
Adela Pérez Galvín ${ }^{1}$, Auxi Barbudo Muñoz ${ }^{2}$, Jesús Ayuso Muñoz ${ }^{3}$, Manuel \\ García Beltrán ${ }^{4}$, Julia Rosales García ${ }^{5}$, Antonio López Uceda ${ }^{6}$
}

Fecha de recepción: 20/10/2019; Fecha de revisión: 25/11/2019; Fecha de aceptación: 04/12/2019

P. Galvín, A., Barbudo, A., Ayuso, J., García-Beltrán, M., Rosales, J., López-Uceda, A. (2019). Introducción al modelado de información de construcción (BIM) en edificaciones e infraestructuras de ingeniería. Revista de Innovación y Buenas Prácticas Docentes, 8(4), 107-115.

\section{Autor de Correspondencia: apgalvin@uco.es}

\section{Resumen:}

La actual exigencia técnica del manejo de programas de diseño en el ámbito de la Ingeniería Civil y la Edificación lleva a plantear de forma indispensable la necesidad de formación de los futuros profesionales en la metodología BIM (Building Information Modeling).

Mediante el presente Proyecto de Innovación Docente se ha desarrollado un modelo de implantación de esta metodología adaptándolo al programa actual de cada asignatura y a las competencias exigidas. La metodología y conocimientos teóricos y prácticos de las herramientas BIM han sido impartidos a 40 alumnos aproximadamente pertenecientes a tres títulos y niveles universitarios diferentes. Los resultados obtenidos muestran una evaluación satisfactoria por parte de los alumnos, respondiendo mediante la enseñanza de nuevas herramientas de diseño a las crecientes necesidades profesionales y enriqueciendo las habilidades de los estudiantes universitarios.

Palabras clave: BIM, Formación, Innovación, Competencias

\section{Abstract:}

The current technical requirement of the management of design programs in the Civil Engineering and Building sector leads to raise the need for training of future professionals in BIM technologies (Building Information Modeling).

Through this Teaching Innovation Project, a model for the implementation of this tool has been developed. The tool has been adapted into the current educational program of each subject and to the required competences. The methodology and theoretical and practical knowledge of the BIM tool have been taught to approximately 40 students belonging to three different university degrees and levels. The results obtained show a satisfactory evaluation by the students. The growing professional needs are met, and the skills of university students are enriched by teaching new design tools

Key Words: BIM, Training, Innovation, Competencies

\footnotetext{
${ }^{1}$ Universidad de Córdoba (España), apgalvin@uco.es; CÓDIGO ORCID: 0000-0001-7494-6200

${ }^{2}$ Universidad de Córdoba (España), abarbudo@uco.es; CÓDIGO ORCID: 0000-0002-6540-2575

${ }^{3}$ Universidad de Córdoba (España), ir1ayuje@uco.es; CÓDIGO ORCID: 0000-0001-5812-678X

4 Universidad de Córdoba (España), manugabel@gmail.com; CÓDIGO ORCID: 0000-0001-7653-5261

${ }^{5}$ Universidad de Córdoba (España), jrosales@uco.es; CÓDIGO ORCID: 0000-0001-6882-1196

${ }^{6}$ Universidad de Córdoba (España), p62louca@uco.es; CÓDIGO ORCID: 0000-0002-4514-9541
} 


\section{INTRODUCCIÓN}

Para cada titulación, la Universidad, a través de sus docentes debe ser capaz de formar en nuevas competencias que doten a los profesionales egresados con una formación que mejore su relación con el ámbito social y empresarial al que se enfrentan (Spinel \& Ortiz, 2003). El presente Proyecto se desarrolla para cubrir la formación básica de docentes en las nuevas herramientas de diseño en ingeniería e introducir a los alumnos en su conocimiento y aplicación.

En los últimos años se ha producido un cambio en el sector de la construcción, por ese motivo, la formación universitaria debe adaptarse a las nuevas tendencias del sector laboral (Becerik-Gerber, Gerber, \& Ku, 2011). Desde febrero de 2014, la Industria de la Construcción Española ha estado sujeta a la Directiva UE 2014/24/UE1, la cual invita a modernizar la contratación pública europea y reducir los costes en los 28 Estados miembros de la UE. Esta directiva permite a los Estados miembros a fomentar, especificar e incluso requerir el uso de Building Information Modeling (BIM) para proyectos de construcción financiados con fondos públicos de la UE a partir de 2016. La formación universitaria de los futuros profesionales para la consecución de los objetivos europeos fijados es muy escasa. Actualmente, la formación académica en la universidad se está esforzando por incorporar paulatinamente la metodología BIM (Cho, Hong, Lee, \& Jang, 2014), en búsqueda de dos avances principales:

1. la preparación para un entorno profesional marcado por esta metodología,

2. la mejora en el aprendizaje global propiciada por "diseño comprensivo" característico del entono BIM (Sharag-Eldin \& Nawari, 2010).

Pero a día de hoy la implantación de conocimientos de BIM en diferentes asignaturas en casi inexistente, actualmente solo el $23 \%$ de los Grados de Ingeniería y Arquitectura de España introducen el aprendizaje de esta herramienta dentro de sus planes de estudio (Santos, 2017). A diferencia del resto del mundo, en el que se puede observar que la metodología BIM ha sido implantada de forma progresiva en los últimos años, siendo un objetivo marcado por parte de las Administraciones Públicas, en EEUU, Australia, Noruega e Inglaterra el uso de BIM es obligatorio en convocatoria de proyectos públicos (Sharag-Eldin \& Nawari, 2010). En Asia, más del 50\% de las constructoras exigen el uso de esta herramienta (AENOR, 2016). Se puede observar el la Figura 1 el grado de implantación en diferentes países y como en Europa se está estableciendo iniciativas público-privadas para su implantación que muestra de forma más clara la necesidad de formar a los futuros ingenieros en esta herramienta. 


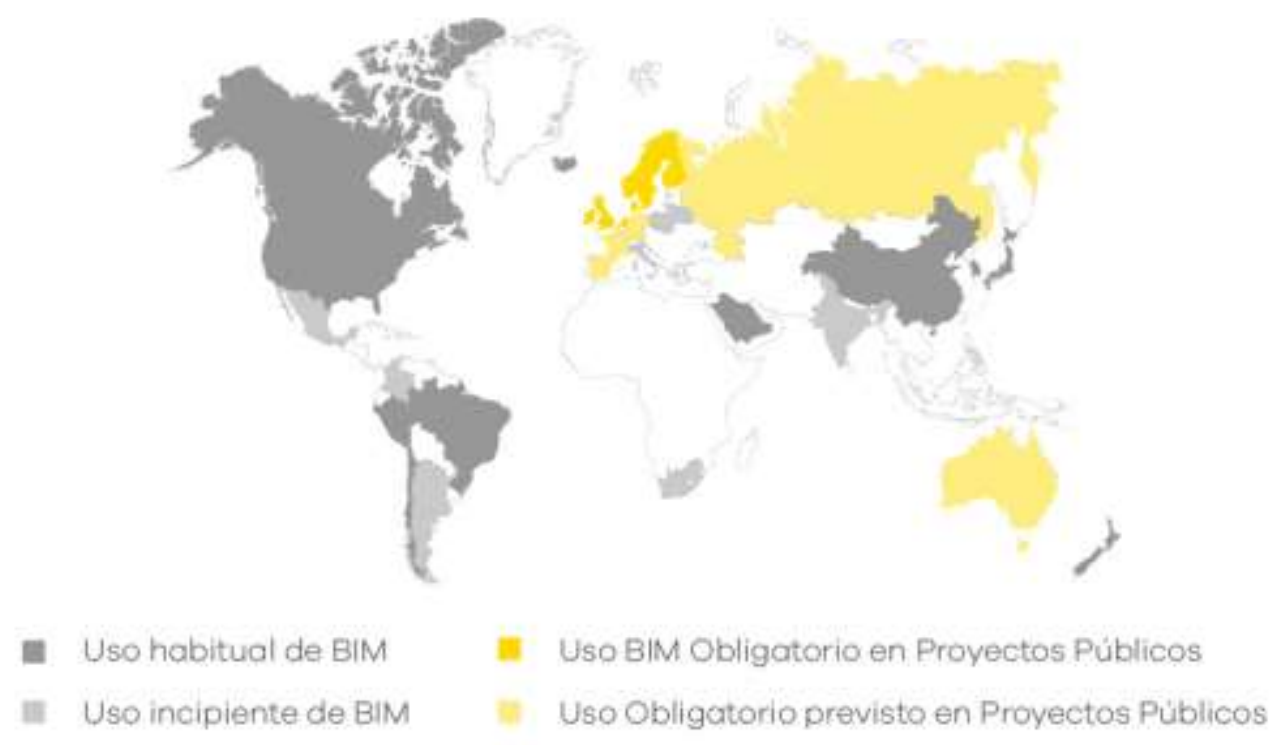

Figura 1. Implantación BIM a nivel mundial, 2016. Fuente: Comisión BIM

Desde el punto de vista docente entendemos que es necesario la incorporación de la metodología BIM a los nuevos estilos de aprendizaje en ingeniería (PiedecausaGarcía, Mateo Vicente, \& Pérez Sánchez, 2015). Como herramienta para uso profesional y como un instrumento TIC idóneo para fomentar la calidad y diversidad de la docencia y un factor de integración y cohesión en el contexto de una estrategia global de desarrollo de competencias del Espacio Europeo de Educación Superior (EEES) (Watson \& Watson, 2007).

Por todo ello, la formación del profesorado en este ámbito para el desarrollo del conocimiento de la herramienta BIM de forma continua en el aula asociada a los conocimientos impartidos actualmente y la aplicación de esta herramienta en proyectos concretos es de gran utilidad para el avance de la formación. El análisis de la implantación de BIM en la universidad y la demanda profesional pone de manifiesto la necesidad de introducir formación BIM en la educación oficial, tanto universitaria como en másteres. En este trabajo se muestran los avances obtenidos mediante el desarrollo del Proyecto de Innovación Docente basado en la formación de los profesores para implementar las asignaturas con nuevos contenidos y actividades asociando BIM a los conceptos de cada asignatura, creando nuevos materiales didácticos que lleven a un mayor desarrollo de los alumnos que han adquirido estos nuevos conocimientos y han aplicado la herramienta de diseño durante el curso de forma presencial y online mediante un continuo seguimiento del profesor que ha hecho posible el logro de nuevos conocimientos y aplicación de nuevas herramientas en el aula.

\section{DESARROLLO DE LA EXPERIENCIA DE INNOVACIÓN}

El objetivo principal que aborda el presente proyecto es que el estudiante adquiera las competencias específicas de Ingeniería de la Construcción recogidas en los actuales planes de estudio para que lo habiliten mejor para el ejercicio profesional. Para la adquisición de estas competencias es necesario el conocimiento de nuevas herramientas informáticas y asociar su uso y aplicación a los conocimientos teórico- 
prácticos impartidos en las asignaturas. Es importante la inclusión de metodologías novedosas de trabajo en el aula que estén estrechamente relacionadas con las exigencias del mercado laboral.

Para conseguir este objetivo principal, se desarrolló una actividad de Innovación planteando los siguientes objetivos secundarios:

- Formar al profesorado en nuevas metodologías de diseño en Ingeniería.

- Introducir al alumnado al modelado BIM mediante la aplicación práctica en diferentes softwares.

- Satisfacer las expectativas del alumnado sobre el diseño práctico de proyectos de Ingeniería.

Para el desarrollo de los objetivos del proyecto se ha seguido la planificación propuesta en tres fases (Figura 2).

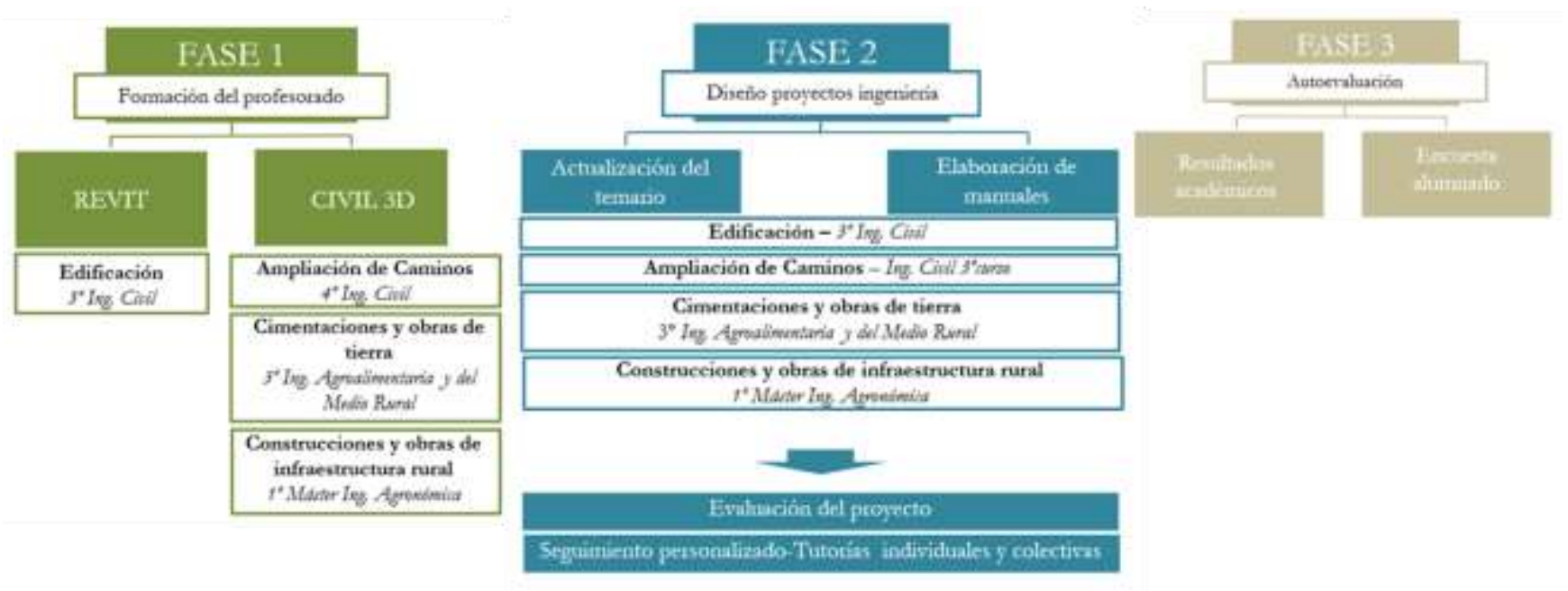

Figura 2. Metodología

\subsection{FASE 1: Formación del profesorado}

La fase inicial del Proyecto ha consistido en la formación del profesorado encargado de desarrollar la correspondiente herramienta BIM en su asignatura. Para ello, cada uno de los participantes en este proyecto han recibido formación específica de la herramienta a desarrollar.

- 1 curso de 25 h lectivas de REVIT ARQUITECTURA (nivel básico)

- 1 curso de $20 \mathrm{~h}$ lectivas de REVIT ESTRUCTURA (nivel básico)

- 2 cursos de CIVIL 3D (nivel intermedio)

De forma simultánea, durante el desarrollo de cada una de las actividades y proyectos en el aula, la colaboración y supervisión entre profesores ha sido continua. Se ha llevado a cabo la elaboración de material didáctico, informes, y encuestas, así como asesoramiento técnico para la impartición de dicha metodología.

\subsection{FASE 2: Diseño de proyectos de Ingeniería con BIM}

Para cada una de las asignaturas reflejadas en la Figura 2 se llevó a cabo el desarrollo práctico mediante herramientas BIM de los conocimientos teóricos adquiridos. Se ha implantado un nuevo modelo de enseñanza-aprendizaje mediante el cual se han desarrollado las siguientes actividades en el aula: 
- Actualización de temario de cada asignatura. Se han seleccionado los conceptos teóricos claves de cada una de las herramientas BIM y se han introducido en el temario existente.

- Se ha iniciado la elaboración de manuales de autoaprendizaje de cada herramienta BIM para que, de forma sencilla, los alumnos puedan seguir de manera autónoma los mismos, sirvan de apoyo para la comprensión de cada software, y para la elaboración de los proyectos. En este aspecto, se pretende profundizar y mejorar en la continuación de este proyecto (ya aprobada) el próximo curso académico.

- Elaboración de ejercicios sencillos entregables durante el curso para llevar a cabo la práctica y el desarrollo de la metodología BIM en la asignatura, en los que se combinan conceptos teóricos, prácticos y resolución de estos mediante herramientas BIM.

- Evaluación del Trabajo de diseño y cálculo (Proyecto). De esta forma se comprueban las competencias adquiridas por los alumnos.

En todo momento, los profesores han estado en contacto directo con los alumnos, estableciendo tutorías colectivas e individuales durante todo el curso, en forma telemática o presencial, para resolver dudas puntuales, revisar actividades, etc. De forma paralela, ha habido una comunicación continua entre los profesores participantes, apoyándose en el manejo del software y organización de las sesiones.

\subsection{FASE 3: Autoevaluación}

Es necesaria la evaluación de los resultados y de la experiencia desarrollada en el Proyecto de Innovación Docente para que de esta manera los profesores desarrollen una autocrítica que refleje si el sistema adoptado para la enseñanza, la metodología, las herramientas, etc., son correctas. Para ello desarrollaron dos sistemas de evaluación:

- Revisión de los resultados académicos, evaluando la puntuación obtenida por los alumnos.

- Consulta al alumnado mediante encuestas de valoración.

\section{RESULTADOS}

De acuerdo con los objetivos propuestos, los profesores han adquirido conocimientos de BIM que no se habían implantado en ninguna asignatura hasta la fecha de hoy. A partir de estos conocimientos, se ha desarrollado un nuevo material didáctico en cada una de las asignaturas sujetas a este Proyecto de Innovación Docente que introduce el conocimiento y manejo de softwares BIM.

La Tabla 1 resume todo el material didáctico nuevo, mediante las actividades que han sido introducidas en cada una de las asignaturas en función a la herramienta BIM a aplicar, así como, las herramientas de evaluación que han sido consideradas en cada caso para comprobar las competencias adquiridas por los alumnos. 
Tabla 1. Actividades desarrolladas

\begin{tabular}{lccc}
\hline & $\begin{array}{c}\text { Herramienta } \\
\text { BIM }\end{array}$ & Titulación & № Alumnos \\
\hline Edificación & REVIT & Ingeniería Civil & 36 \\
\hline $\begin{array}{l}\text { Ampliación de } \\
\text { Caminos }\end{array}$ & CIVIL 3D & Ingeniería Civil & 9 \\
\hline $\begin{array}{l}\text { Cimentaciones y } \\
\text { Obras de Tierra }\end{array}$ & CIVIL 3D & $\begin{array}{c}\text { Ingeniería Agroalimentaria y del } \\
\text { Medio Rural }\end{array}$ & 11 \\
\hline $\begin{array}{l}\text { Construcciones y obras } \\
\text { de infraestructura rural }\end{array}$ & CIVIL 3D & Máster Ingeniería Agronómica & 43 \\
\hline
\end{tabular}

\subsection{Resultados académicos}

Los resultados académicos, tal y como era de esperar, no mostraron correspondencia con el grado de satisfacción del alumnado (apartado 3.2.).

El objetivo de este proyecto de innovación no consistía tanto en aumentar la tasa de aprobados en primera convocatoria, sino mejorar la formación del alumnado en el manejo de herramientas informáticas que van a necesitar manejar en su actividad profesional al finalizar sus estudios.

\subsection{Encuestas-Valoración}

En la propuesta elaborada se establecía que uno de los objetivos básicos para la evaluación del Proyecto era satisfacer las expectativas del alumnado. Para la consecución de este objetivo se lleva a cabo una valoración final de las actividades implementadas por parte de los alumnos mediante encuestas. Para ello, un total de 40 alumnos fueron encuestados.

El análisis de estos datos en cada una de las asignaturas es útil para seguir implementado la metodología BIM de forma similar o por el contrario modificar la forma de implantación, corregir errores en la metodología establecida o constatar la correcta aplicación y valorar el aprendizaje y la aceptación del alumnado.

En la Figura 3 se muestra una captura de una de las encuestas elaboradas para recabar la opinión de los alumnos, diseñadas entre todos los profesores, para obtener, de la forma más detallada posible, la opinión de estos, y así, poder obtener conclusiones. 


\section{EPSB}

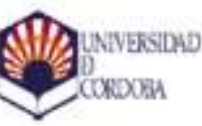

\section{"INTRODUCCIÓN AL MODELADO DE INFORMACIÓN DE CONSTRUCCIÓN (BIM) EN LA ASIGNATURA DE EDIFICACIÓN" ENCUESTA DE SATISFACCIÓN}

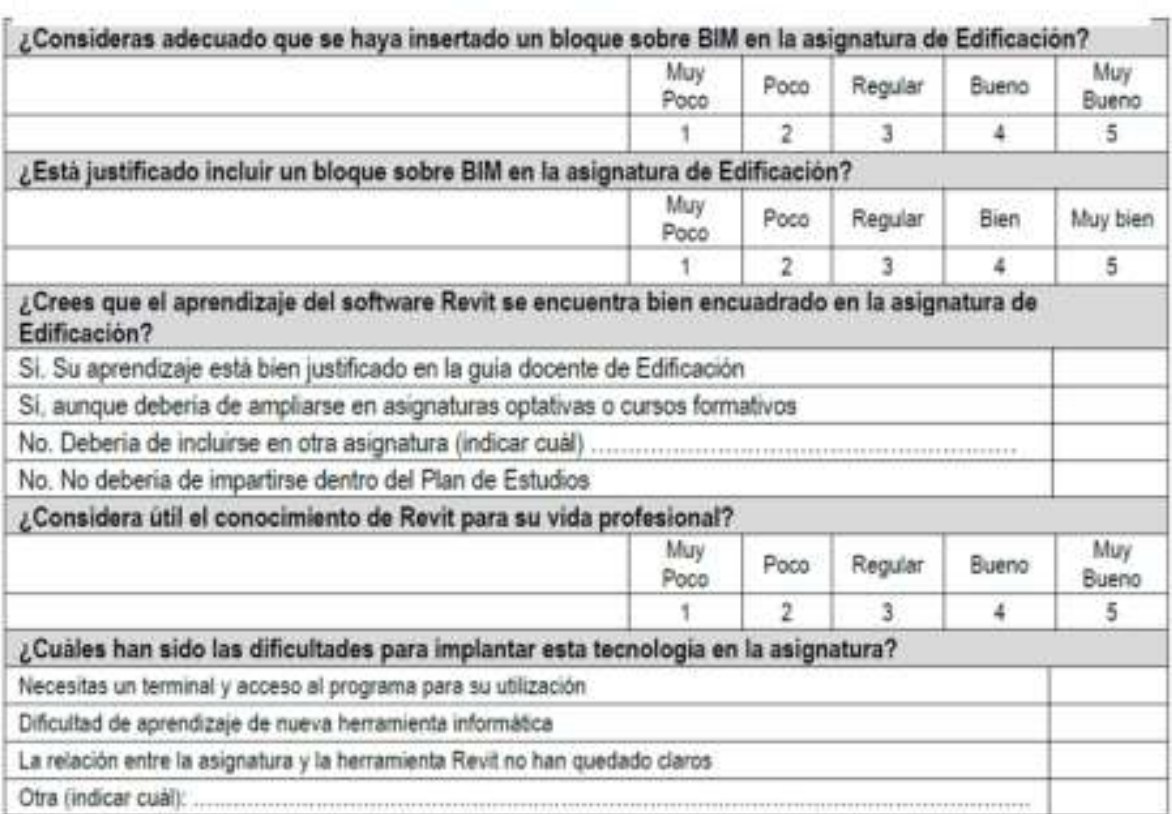

Figura 3. Muestra de encuesta de satisfacción del alumnado.

En relación a la opinión de los alumnos respecto a cómo está encuadrada en las diferentes asignaturas la herramienta BIM impartida (Figura 4), se puede apreciar que prácticamente la totalidad de los alumnos mostraron una respuesta afirmativa, indicando que debería ampliarse la oferta formativa con asignaturas optativas para la docencia de las herramientas BIM impartidas u otras actividades formativas. 


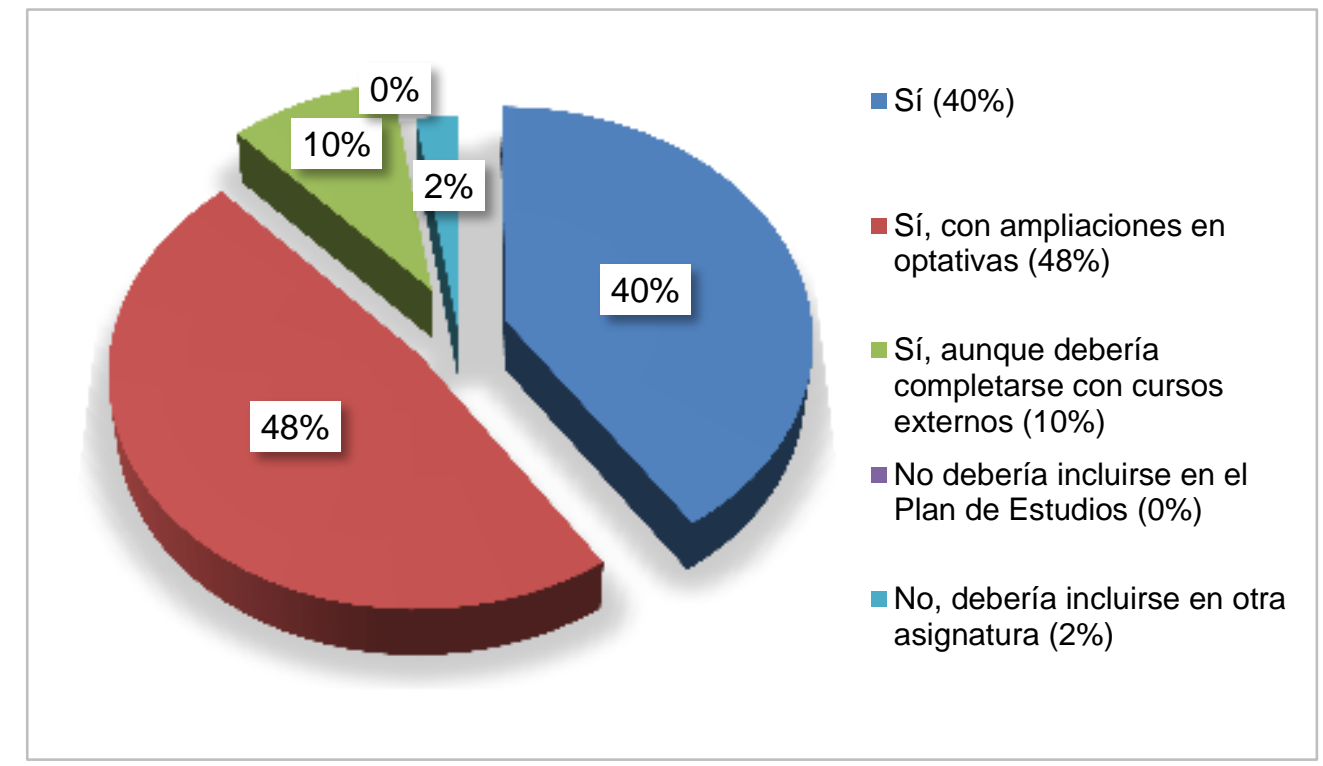

Figura 4. Distribución porcentual de respuestas sobre el encuadre de la herramienta BIM en cada una de las asignaturas.

En relación con la opinión de los alumnos respecto a si consideran útil la herramienta BIM impartida (Figura 5), se puede apreciar que prácticamente la totalidad de los alumnos, mostraron una respuesta positiva, sobre todo, para su futuro profesional.

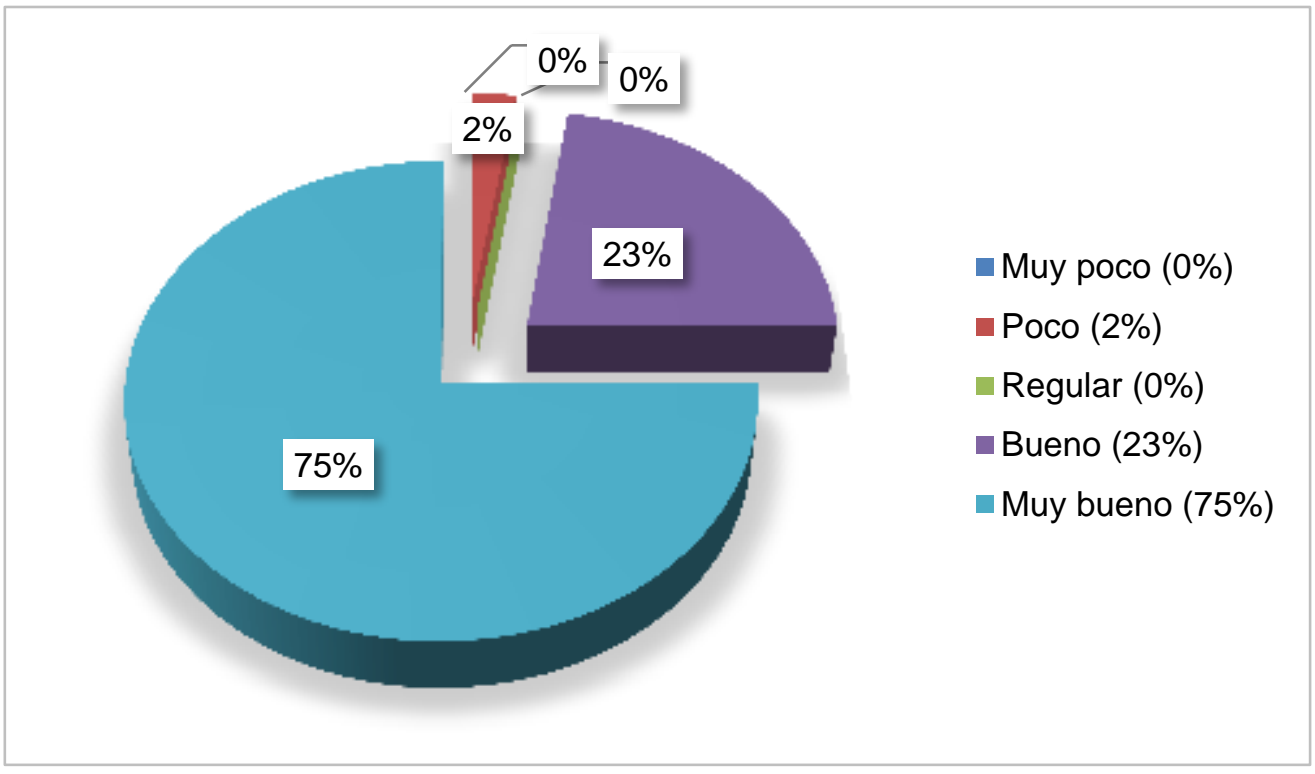

Figura 5. Distribución porcentual de respuestas a la pregunta: "Considera útil el conocimiento de la herramienta BIM impartida para su vida profesional" en las tres asignaturas.

Además, en respuesta a otros comentarios relativos a la impartición de la herramienta BIM, algunos alumnos señalaron que el manual de apuntes era susceptible de ser mejorado.

Como resumen de la autoevaluación podemos considerar que los resultados obtenidos en cada una de las tres asignaturas respecto a cada software BIM aplicado han sido similares. Los alumnos consideran mayoritariamente (aproximadamente $90 \%$ de los encuestados) un software muy adecuado para la asignatura, así como muy útil 
a nivel profesional (aproximadamente un $80 \%$ de encuestados). El $100 \%$ de los encuestados valora de forma general buena o muy buena el software estudiado.

Respecto a la metodología seguida en el aula, cabe destacar que el $30 \%$ de los encuestados consideran que el aprendizaje de la herramienta presenta ciertas dificultades y que el volumen de trabajo es elevado (78\%) para la cantidad de clases impartidas, considerando el $50 \%$ de los alumnos encuestados regular el número de clases.

Como visión general, la valoración por parte de los alumnos muestra la inclusión de la herramienta BIM en el aula como útil y necesaria a la vez que refleja la insuficiencia de clases para impartir todos los conocimientos.

\section{CONCLUSIONES}

A la luz de los resultados relativos a las consultas realizadas al alumnado mediante encuestas de valoración para la introducción al modelado de información de construcción (BIM) en asignaturas relativas a edificaciones e infraestructuras de ingeniería, podemos resaltar que:

- Las horas impartidas de cada herramienta BIM correspondiente a cada asignatura han sido muy valoradas por los alumnos, pero atendiendo a sus respuestas, todavía insuficientes. Los alumnos proponen mayoritariamente que las herramientas BIM sean ampliadas en optativas o en otras actividades extra-académicas.

- Se sugiere, para el año que viene, una mejora y ampliación del manual de apuntes como guía de orientación para el trabajo no presencial.

- La incursión de la metodología BIM como nuevo estándar en el sector de la construcción en todas las fases del proceso constructivo conlleva una revolución total a todos los niveles el sector. Es por esto por lo que la Universidad tiene el deber de evolucionar no sólo a la par, sino ser referente en investigación y desarrollo, implicando para ello cambios significativos en los procesos de enseñanza y aprendizaje del alumnado.

\section{REFERENCIAS}

AENOR. (2016). Estándares en apoyo del BIM. Informes de Normalización.

Becerik-Gerber, B., Gerber, D. J., \& Ku, K. (2011). The pace of technological innovation in architecture, engineering, and construction education: integrating recent trends into the curricula. Journal of Information Technology in Construction (ITcon), 16(24), 411-432.

Cho, Y. S., Hong, S. C., Lee, J. H., \& Jang, H. S. (2014). Higher education program development for structural building information modeling (S-BIM). Paper presented at the Advanced Materials Research.

Piedecausa-García, B., Mateo Vicente, J. M., \& Pérez Sánchez, J. C. (2015). Enseñanza de sistemas BIM en el ámbito universitario.

Santos, A. G. (2017). Mapa de la formación BIM en la Universidad

Sharag-Eldin, A., \& Nawari, N. O. (2010). BIM in AEC education. Paper presented at the Structures Congress 2010.

Spinel, S. C., \& Ortiz, J. C. R. (2003). Prácticas docentes que promueven el aprendizaje activo en Ingeniería Civil. Revista de ingeniería, 18, 48-55.

Watson, W., \& Watson, S. L. (2007). An argument for clarity: What are learning management systems, what are they not, and what should they become. 